\title{
Thoughts on the Implementation of Problem-based Learning Methodology in Biochemistry Teaching
}

\author{
Xiaojia Hu, Kaihua Long, Liang Li \\ Xi'an Peihua University, Shaanxi, Xi'an 710125, China
}

\begin{abstract}
Keywords: Problem-based, Teaching Method, Biochemistry, Application, PBL.
\end{abstract}
\begin{abstract}
PBL (Problem-based Learning) teaching method is a teaching method that based on the problem, which can stimulate students' interests in learning and practicing their ability of thinking. Biochemistry is one of the foundation courses in colleges and universities, and the PBL teaching method can achieve better results. This article, from the features of PBL teaching method and biochemistry, analyzes the necessity for the application of PBL teaching method in biochemistry teaching, discusses the application of PBL teaching method in the teaching of biochemistry process, and discusses the PBL teaching method on further application in biochemistry teaching.
\end{abstract}

\section{The Necessity of Implementing PBL Teaching Method in Biochemistry Teaching.}

\subsection{The Definition and Characteristics of Biochemistry}

Biochemistry is a subject that uses the theory and method of chemistry, from the molecular level, to study the rules of chemical changes of the chemical composition of the organism in the process of life. Biochemistry is also the foundation course of biology technology, applied chemistry, food engineering majors, etc., which is an especially important foundation course in medical schools. As a subject developed from physiology and organic chemistry, the teaching content of biochemistry mainly involves the basic structure material of organism, material and energy conversion in life process, etc. The features of the course include rigorous curriculum system, abstract theories, complex and closely tangled knowledge network, etc. As the development and breakthroughs of biochemistry are always accompanied by the scientific and technological progress of the technology, the biochemistry theory and technology have already been implemented in almost every branch of modern medicine field. Therefore, biochemistry is a difficult subject in all related majors.

With the deepening of the medical science, we have more thorough understanding of the proliferation and death, growth and development, genetic variation of the cells, and even of the process such as the origin and evolution of life, thus biochemistry and medical science are closely linked than ever. People have a deeper understanding of past problems, especially genes and genetic diseases. Biochemistry can also help clinical medicine to discuss the etiology, elucidate mechanism, and make diagnosis and control in the molecular level, so how to strengthen the connection between theory and practice, to integrate the foundation knowledge of biochemistry and un-solving clinic problems together, to visualize complex metabolic mechanism to the easy-to-read knowledge, and to cultivate all-round and qualified talents, is the ongoing topic in biochemistry teaching.

\subsection{The Definition and Characteristics of PBL Teaching Method}

PBL (problem-based learning) is a teaching method which takes students as the main body of teaching and takes the problem as the core of teaching to conduct induction and discussion. Compared with the traditional teaching method in which the teacher crams and the students listen, PBL teaching method bears the main objective of cultivating the self-learning ability of students. conducted by the teacher according to the teaching plan, PBL teaching method extracts several key points, provides students with the platform of pondering and solving problems by raising key problems to induce students' consciously learning, communication, and solutions. The focus of PBL teaching is to enable students to learn relevant knowledge and learn methods in problem-solving. Through PBL teaching, students can open their minds; and in the process of solving problems, they can learn relevant knowledge and broaden their vision. Students need to translate the problem-solving process into their 
own language, which helps to break down and reprocess the knowledge, so that students can grasp the knowledge points more deeply. The problem is put forward based on previous knowledge, the student wants to solve the problem, should review the knowledge that has already been studied, to be able to learn something new. Students also can improve their communication skills in communicating with teachers and classmates.

\subsection{The Application of PBL Teaching Method in Biochemistry Teaching.}

At present, many medical schools in China have realized that the traditional teaching mode has not adaptable enough to fit the current medical education development trends and tried to carry out the PBL teaching reform. For example, Bengbu medical school has conducted a trial of PBL teaching mode in many courses such as medical physiology, medical immunology, histology and embryology, and achieved good results. Meanwhile, the PBL teaching method in practice has its own deficiencies. For instance, PBL teaching method requires higher quality of teaches, and many teachers have the misunderstanding of PBL teaching method, mistakenly equals PBL to asking questions as many as possible. Others deficiencies includes poorly-raised questions, irrelevant questions, and the inflexible teaching. Some students have been used to passively accept the knowledge instead of actively finding solutions when encounter questions; they lack the ability of self-learning and cannot catch the key points in the learning process; they do not have the effective study skills, adaptable learning methods, or proper operating skills in practice to help them solve the problems efficiently, nor do they have the proper level of self-control and strong time-management skills. Some of them have been found plagiarizing in the process of solving problems.

\section{The Application of PBL Teaching Method in Biochemistry Teaching}

\subsection{The Selection of Teaching Content}

The content of biochemistry course is rich, making it difficult to carry out PBL teaching in all content under the same class hours. Therefore, it is necessary to choose the key content to carry out PBL teaching. The key and difficult points of biochemistry are material transformation and energy conversion. As the metabolic center organ of the human body, the liver plays an irreplaceable role in the metabolism of organisms. The pathology, physiology and other pathological changes of liver can reveal large amounts of metabolic abnormalities; The clinical diagnosis of liver disease involves the detection and analysis of various biochemical indexes; The clinical treatment plan covers many knowledges of pharmacology and biochemistry and has the characteristics of interdisciplinary integration. Therefore, PBL teaching can be carried out in the teaching of three major substances in metabolism courses.

\subsection{The Selection of Cases}

The case is the platform of PBL teaching in biochemistry teaching, the basis for raising questions and solving problems, and the key to stimulate students' interest in learning. The main contents of the case were age, gender, history, symptoms, clinical diagnosis, test results, etc. Teachers need to combine the teaching content and clinical data, design the cases that meet the teaching goal, that are common in clinical situations, and that are easy and practical for students to understand, which reflects the importance of teaching contents in the theory applications. For example, in the teaching of "sugar metabolism" chapter, teacher can design diabetes, hyperglycemia, hypoglycemia as cases; In the chapter of "metabolism of ammonia", teacher can choose hepatic encephalopathy as cases; In the chapter of "the generation and utilization of ketone bodies", teacher can choose the pregnant women with vomiting during pregnancy as cases.

\subsection{The Problem Design}

The problem is the key of PBL teaching, which is directly related to the teaching effect of biochemistry. We can achieve our purpose that let the students to solve their problem actively and understand the knowledge by Setting up a difficult problem, taking full use of the student's curiosity and arousing the student's learning motivation and the positive thinking. When designing a problem, the teacher must consider the following conditions: first, the design of the problem should be based on 
the teaching content and drawn out the key and difficult points in the course. For example, the "protein" section focuses on "the relationship between protein structure and function". Teachers can use the "the relationship between structure of hemoglobin and the sickle cell anemia". as a problem. After describing the primary structure and advanced structure of proteins, and the pathogenesis of sickle-cell anemia, teachers should organize students to discuss and solve this problem; Second, the question should contain the content of the before and after the chapters in the text, the difficulty should be appropriate, we can't let students easy to find the answer directly, should have certain challenges, so that the students can learn the knowledge step by step, get the interest to solve the problem constantly. For example, the teacher can ask questions about the next chapter after finished the previous chapter, let students preview and study with questions. In the following teaching, the teacher asks students to tell the information they have found. Third, the problem should be able to make teaching content more systematic, teachers should pay attention to combine the relevant knowledge in the design, strengthen students' understanding of knowledge, reflect the inner link and the biological chemistry course system structure, and it should conform to the law of students learning process. For example, in the chapter teaching of "sugar metabolism", the main knowledge points are glycolysis and tricarboxylic acid cycle, etc., the teacher can take "eat candy can make people fat?" as a question, in the case of "fermentation mechanism of food" and "physical pain after vigorous exercise", this is a topic that students are interested in. Students can better understand the process of glycolysis when analyzing the metabolic pathways of cases. Body fat involved in glucose metabolism, amino acid metabolism, fat metabolism, nucleic acid metabolism, almost the entire biochemical knowledge, students can realize the relationship between the biological metabolism of the components, set up knowledge framework, biochemistry make biochemistry learning system and organized.

\subsection{Group Discussion}

Group discussion is an important part of PBL teaching, which can improve the efficiency and quality of problem analysis, especially in biochemistry. Based on students' self-study, the teacher divides students into groups, and the group members communicate and inspire each other, and the process of discussion is also an in-depth process of knowledge learning. When dividing the students into groups, the teacher must divide them equally according to the learning level of members, let each member have their advantages. Teachers should also consider the grade, gender, students intentions, etc., avoid form curing, make students with different character learning together to stimulate their innovation. Students with different degrees of self-study may have differences in opinions. Through discussing, they can find new problems. If they have different views and disputes, they can consult teachers in time. Teachers should also participate in group discussions, participate in the group atmosphere actively, so that teachers can prevent individual students from working in a negative way, and to master the degree of solution to the problem. A relaxed and active group discussion can give full play to the students' intelligence level, the members work together, find the literature in the spare time, find the answers, can strengthen the cooperation ability.

\subsection{Class Discussion}

Classroom discussion is an important embodiment of teachers' leading role and an important way for students to strengthen their thinking ability. Teachers can take out a certain class, organize regular students in group discussion and answers show, each group choose delegates to speak. In the process, teacher should evaluate students' presentation, points out mistakes and shortcomings and puts forward a new question. Teacher can organize students to discuss again according to the speech situation, through the series question, lead the student to develop the ability to use knowledge to solve biochemistry problems. For example, in the teaching of "lipid metabolism", we can take "dietary prevention, drug treatment and weight loss method of fatty liver, obesity and other fat metabolic disorders" as a problem. After students summarize the information and puts forward the solution, in the class discussion, the team can take a form of speech, such as oral reports, PowerPoint presentations. And then Teachers should review and summarize group work, guide the student to review the solving process. 


\subsection{Summary after Class}

After the problem solving, the teacher should let students submit a summary report in form of every student or every academic group. The summary content could be in the form of a written report or PPT, including questions, selecting case, difficulties and solving methods, so the students can strengthen and consolidate the knowledge. The teacher also should write the teaching report according to the PBL teaching process, and record the problems existing in the process of solving the problem and adjust next time. Teacher should give students score according to their report and classroom performance. The score should be record in the biochemistry course grade [4]. What's more, the teacher should also summarize students feedback like their acceptance and satisfaction with PBL teaching, case selection, design of question, organizational forms, listen to the students actively, promote the further application of the PBL teaching method in the teaching of biochemistry.

\section{Conclusion}

To sum up, PBL teaching method can stimulate students' interest in learning and improve students' ability to solve problems and practice theory. The PBL teaching method has achieved good teaching effect, it has high acceptance among students and has been widely recognized by the educational circles. At the same time, the PBL teaching method has an high requirement on teachers thought concept, knowledge reserve, teaching skills, the students' self-study ability, self-discipline and efficient teaching resources, teaching evaluation ability. This cause some difficulties in practical application. Therefore, to realize the further application of the PBL teaching method in the teaching of biochemistry, we need to improve the teachers' quality, deepen education reform, improve the teaching evaluation system, teaching students according to their actual circumstance. At the same time, we should explore new teaching methods based on PBL, for example, we can combine case teaching method (case-based learning, CBL) with PBL teaching method [5], or the combine PBL teaching method with network and computer technology to new teaching method.

\section{References}

[1]. Ma Jia, Chen Changjie, Xia Jun, et al. Discussion on the reform of medical biochemical case teaching [J]. Basic medicine education, 2011,13(7):613-615.

[2]. Guo, Yang Qingling, Ma Jia, Zhou Jihong, Shi Yurong, Lu Jingzhu, Liu Changqing, Xia Jun. Application of PBL teaching method in biochemistry teaching in medical colleges [J]. Basic medicine education, 2013,15(04):341-343.

[3]. Lu Huiru, Li Jiaofeng, Cui Yanhong, Wang Nali, Guo Jinxiang. Application of PBL teaching method in the biochemistry teaching of adult teaching class [J]. Development of modern biomedical science,2012(08):1560-1562.

[4]. Cheng Shan, Niu Jing, Yu Hefen, Ding Wei. Practice of PBL teaching in basic medical biochemistry teaching $[\mathrm{J}]$. Basic medicine education,2017(06):415-418.

[5]. Jiang Zhou, Yao Xuzhou, Zhu Peifang, Chen Haifeng. Introduction of PBL teaching method in biochemistry experiment teaching and development trend $[\mathrm{J}]$. Journal of education college,2015(02):114-115. 